\title{
Children's Imaginaries of Robots for Playing With
}

\author{
Adriana Maria Ríos Rincón ${ }^{1}$ (]) William Ricardo Rodríguez-Dueñas ${ }^{2}$ (D) Daniel Alejandro Quiroga Torres ${ }^{2}$ (1) \\ Andrés Felipe Bohórquez ${ }^{2} \cdot$ Antonio Miguel-Cruz ${ }^{1,3,4} \mathbb{E}$
}

Accepted: 15 June 2021 / Published online: 25 June 2021

(c) The Author(s) 2021

\begin{abstract}
Children with severe motor impairment due to cerebral palsy have difficulties engaging in play, although they want to play games that typically developing children play. The barriers imposed by motor impairments against engaging in play can be addressed through the use of robots. We aim to identify how children, who have extensive experience of play, imagine what a robot is and what features would make a robot good to play with. Using a qualitative description design, 19 children from urban and rural settings participated in focus groups to draw and talk about the robots they would like to exist. The data were coded and analyzed using a summative approach to content analysis. The findings revealed that the children imagined that a good robot to play with is one that has an anthropomorphic appearance, is tough and strong, has controls, and that is able to move, grab, speak, and play popular children's games. In particular, the girls imagined that robots should be able to express positive emotions towards children. Age, gender, culture, and the physical environment in which the children lived influenced what they expected to find in a robot for playing with and how they imagined child-robot interactions.
\end{abstract}

Keywords Robot $\cdot$ Children $\cdot$ Physical disabilities $\cdot$ Cerebral Palsy $\cdot$ Play

\section{Introduction}

The literature on play consistently asserts that play is critical for children's development [1]. Play is an ideal way for children to discover the world and learn through practice with different objects and experiences [2]. Similarly, the literature in the field of rehabilitation consistently asserts that children with severe motor impairment due to cerebral palsy have difficulties engaging in play [3]. Children with cerebral palsy have motor control problems that affect their gross and fine motor skills, and, in turn, their manipulation. Without

Adriana Maria Ríos Rincón

aros@ualberta.ca

1 Department of Occupational Therapy. Faculty of Rehabilitation Medicine, University of Alberta, 2-64 Corbett Hall, Edmonton, AB T6G 2G4, Canada

2 School of Medicine and Health Sciences, Universidad del Rosario, Calle 63D \# 24-31, 7 de Agosto, Bogotá, D.C, Colombia

3 Glenrose Rehabilitation Hospital, 10230111 Ave NW, Edmonton, AB T5G 0B7, Canada

4 Faculty of Health, University of Waterloo, 200 University Ave W, Waterloo, ON N2L 3G5, Canada manipulation, children's play is less effective for exploring their environment and for learning from the properties of objects, thus making it difficult for those children to learn and develop because they cannot interact with the environment in an effective way. As a result, they miss many opportunities to develop the motor, social, emotional and cognitive skills that typically developing children naturally develop through play. Consequently, the senses of self-efficacy and self-determination are affected in children with cerebral palsy and their development is delayed [4].

Robots have shown the potential to assist children with cerebral palsy in engaging in play. In the last 5 years, published surveys have synthesized the knowledge about the research and commercial availability of such robots. In their systematic literature review, van den Heuvel and colleagues [5] explored the aims, control options, and commercial availability of robots to support play in children with severe physical disabilities, and found eight robots reported in 19 studies, none of which were commercially available. Miguel and colleagues [6] examined the extent and types of robots used to promote play and development in children with cerebral palsy and found six robotic systems reported in 14 studies. The findings of this systematic literature review revealed that most robots used 
for interventions were commercially well-established and required technical support personnel for adaptation, configuration, and troubleshooting. Also, a very small number of robots have been developed that can be used with children with motor impairments; these robots are still at the prototype stage and have only been used in controlled environments. These two systematic reviews provide insights that: (1) children with severe motor impairment want to play games that typically developing children play, but they do not have the motor skills to perform these activities; (2) the barriers imposed by motor impairments against engaging in play can be addressed through the use of robots; (3) no robots are currently readily available to be used by therapists, families, caregivers or educators of children with motor impairments to promote engagement in play; (4) most of the robots used in previous studies were adapted so that children controlled them using different body parts such as their upper limbs, head or lower limbs (e.g. Lego ${ }^{\mathrm{TM}}$ and PlayROB); (5) some robots detected the children's movements and reacted to those movements (e.g. Cosmobot, IROMEC, and AIBO) so those robots acted as toys; and (6) only a few robotic systems were designed or adapted to interact with objects and toys in the environment (e.g. reaching for and grasping objects) in order to compensate for the limitations the children with cerebral palsy had in reaching and grasping due to their motor impairment. These robotic systems acted both as toys and tools to interact with other toys (i.e. $\mathrm{Lego}^{\mathrm{TM}}$, PlayROB, an adaptation of the CRS A465, the Handy- 1 and the Lekbot).

In addition to these literature reviews, recent studies have provided evidence that children with severe motor impairment due to cerebral palsy who had an adapted Lego $^{\mathrm{TM}}$ robot that they controlled using switches demonstrated enhanced levels of playfulness. However, the Lego $^{\mathrm{TM}}$ robots showed some limitations as some children expressed frustration when the robot was not able to perform some of the play activities they wanted to do such as grasping or pushing toys of different sizes and shapes [7]. The children's mothers suggested that the robot could have been bigger, that it could have had grips for grabbing toys and that it could have been more robust as some of its pieces fell off, such as a scoop when the robot was pushing some toys, which disrupted the children's play in some way [8]. Bulgarelli and colleagues [9] investigated the engagement and playfulness of children with severe motor impairment due to cerebral palsy when interacting with five mainstream robotic toys and found that the children engaged in playful interactions with the robots when the children had control over the robots' actions. Thus, from the perspective of children with motor impairment and their families, current research shows that when a robot has features that allows a child to control it, the robot supports play in this population. However, such a robot is not yet commercially available.

\subsection{Developing Robots for Children With Motor Impairment}

When developing robotic systems for children with disabilities, the most common practice is to consult with experts who are usually therapists or educators with clinical experience in providing treatment to children with disabilities. Mahdi and colleagues [10] consulted a children's special needs expert to design a social robot for robot-assisted play for children with motor impairment. Clark and colleagues [11] developed a semi-autonomous socially assistive robot that acted as a toy to facilitate engagement in play and found that subject-matter experts, in this case pediatric therapists, reported that children were engaged in interacting with the robotic toy. Marti and colleagues [12], in a user-centered design approach for the development of the IROMEC robot, asked teachers, special educators, parents, care-givers, and therapists for ideas to elaborate on the robot's requirements. Researchers also observed children while they were playing in order to gather insights about those children's needs. Kronreif [13] and colleagues interviewed therapists and parents of children with disabilities during the process of designing the PlayROB system, a robot that assists children with motor impairment with playing with $\mathrm{Lego}^{\mathrm{TM}}$ bricks. None of these studies developed user requirements from a child's perspective by asking children about what they would like to find in a robot for playing with.

\subsection{Child-Robot Interaction and Its Imaginaries}

One topic that has become prominent in the field of designing interactive and assistive robots is child-robot interaction, which comprise human-robot interaction when the humans are children. In a recently published literature review, de Jong and colleagues [14] pointed out that the acceptance of social robots by children determines the child-robot interaction and that research in this area is still in its early stages. The authors found that a robot's appearance, social behaviour, and adaptive behaviour seem to have an effect on children's acceptance of social robots, although this effect remains inconclusive. Studies in this area that have investigated children's acceptance of a particular robot or a few robots and their interactions have commonly been constrained by the research designs (e.g. the time of an interaction with a robot or a scripted child-robot interaction). Other researchers have found that design choices such as material, size, and degree of mobility influence children's acceptance of robots [15].

In their narrative literature review, van Straten and colleagues [16] identified two critical dimensions for 
child-robot relationship formation; the robot's characteristics (e.g. responsiveness, expressiveness, and tailoring of functionality) and its styles of interaction (e.g. strategic interactions and expression of emotions). The authors found that children perceived a robot as being a social, humanlike being, and that this perception may influence the formation of the child-robot relationship. Playing with robots led to more closeness, enjoyment, and positive affect than playing with inanimate objects. The authors also pointed out that research into child-robot relationship is still in its early stages.

Vallès-Peris and colleagues [17] pointed out that the child-robot interaction contains imaginaries of relationships. An imaginary is a representation or a symbol through which humans imagine objects using past impressions and current knowledge [18]. In technology, the concept of the imaginary has been used to explain how technological advances are influenced by the social and cultural imaginaries of the future and constrained by the current conditions of scientific development [19]. With regard to social robots, the creative process seems to be in the hands of a small group of people (e.g. researchers and companies), which means that the predominant approach is that the final users have to adapt to any innovations [20]. Another emerging approach is to involve the final users in the process of shaping social robots [17].

Although children can adopt different roles in the technology design process such as users, testers, informants or design partners [21], current knowledge about child-robot interaction and the acceptance of robots by children has been built predominantly involving children as users or testers of specific robot models included in studies. Only a few studies have explored children's imaginaries of social robots in a few settings including healthcare [17] and education [22].

\subsection{The Present Study}

Although children are "experts" in play, to the best of our knowledge no studies have investigated children's imaginaries of a robot for playing with. Thus, it still unknown what features children would like to find in a robot for playing with from a child's perspective. We believe that children are "experts" in play and that typically developing children with extensive play experience can tell us what features and appearance a robot for playing with should have. As research has shown that children with motor impairment want to play the same games that typically developing children play, but cannot do so due to limitations in movement, in our study we decided to study the imaginaries of typically developing children. The literature has also reported that children with disabilities such as cerebral palsy have fewer opportunities to play [3]; thus, they have limited play experience [7]. Our study informs on the design of robots for promoting engagement in play in typically developing children, which can be eventually transferred to the design of robots for play for children with motor impairment. Based on this, the present study's research question is: How do typically developing children imagine what a robot is and what features would make a robot good for playing with?

\section{Method}

\subsection{Study Design}

We felt that the best method for addressing our research question was a qualitative approach as we wanted to "hear" the children's voices. This study used a qualitative approach, a qualitative description design [23], and responded to a post-positivist research paradigm as it supports methodological pluralism and is based on the assumption that the selection of the method to be applied in a particular study depends on the research question being addressed [24]. In this study, we followed the Standards for Reporting Qualitative Research (SRQR) [25].

\subsection{Researchers' Characteristics and Reflexivity}

The main author (AMRR) of this study is a woman occupational therapist with more than 10 years of experience working with children with cerebral palsy and conducting research in assistive technologies. AMRR has a PhD degree and led all the focus groups. AMC, WRRD, DAQT and $\mathrm{AFB}$ are all men and engineers involved in research into the development and testing of technologies for health and rehabilitation. AFB is a mechanical engineer research assistant in this project. AMC and WRRD have PhDs, while DAQT and AFB have bachelor degrees. None of the researchers had any previous relationship with the children who participated in the study or with their parents. The recruitment material stated that the researchers were investigating what features children would like to see in a robot for playing with. Boys and girls were invited to attend a 2-h session where they were going to draw a robot that they would like to play with and then tell the researchers what they would like that robot to do.

\subsection{Context}

This study was conducted in a low-middle-income country. The data collection was carried out in two settings. One site was a classroom in a higher educational level institution located in an urban environment in Bogotá, Colombia. This setting was not familiar to the participants, who were invited to attend a focus group at the weekend. The second site was a school located in a small town (Vereda Bagazal, Villeta, Colombia) in a rural environment. In this setting, the 
Table 1 Description of each data collection stage

\begin{tabular}{|c|c|c|c|}
\hline Stage & Objectives to be achieved with the children & Activities done by the children & Questions asked to the children \\
\hline Asking & $\begin{array}{l}\text { To think and verbally communicate the } \\
\text { children's knowledge of and previous } \\
\text { experience with robots }\end{array}$ & $\begin{array}{l}\text { The children responded to questions in a } \\
\text { group session }\end{array}$ & $\begin{array}{l}\text { Have you ever seen a robot? } \\
\text { Do you know what a robot is? } \\
\text { What does a robot do? } \\
\text { What can you do with a robot? } \\
\text { What do you like most about robots? } \\
\text { Have you ever played with a robot? } \\
\text { What did you like most about playing with } \\
\text { that robot? } \\
\text { Would you like to play with a robot? } \\
\text { Are any of your toys robots? } \\
\text { Are any of the toys of someone you know } \\
\text { robots? } \\
\text { What are the names of those robots? } \\
\text { What are those robots like? }\end{array}$ \\
\hline Drawing & $\begin{array}{l}\text { To imagine the robot the children want } \\
\text { To choose the robot's appearance and } \\
\text { functionality }\end{array}$ & $\begin{array}{l}\text { Individually but working on shared work } \\
\text { stations, the children drew their robots }\end{array}$ & $\begin{array}{l}\text { If we told you that we were going to build } \\
\text { a robot to play with, how would you like } \\
\text { that robot to be? } \\
\text { Is there anything you would like that robot } \\
\text { to have? } \\
\text { Is there anything you would like that robot } \\
\text { to do? }\end{array}$ \\
\hline Showing & $\begin{array}{l}\text { To associate the robot's appearance and its } \\
\text { functionality }\end{array}$ & $\begin{array}{l}\text { Each child presented his or her robot to the } \\
\text { group of children } \\
\text { The children were allowed to comment on } \\
\text { the other children's robots }\end{array}$ & $\begin{array}{l}\text { Tell us about your robot } \\
\text { What can your robot do? } \\
\text { How big would your robot be? } \\
\text { What material would your robot be made } \\
\text { of? } \\
\text { How fast would your robot be? } \\
\text { What games could your robot play? } \\
\text { Additional questions about a specific robot's } \\
\text { appearance and components (e.g. what is } \\
\text { this little button for?) }\end{array}$ \\
\hline
\end{tabular}

researchers travelled to the school, which was familiar to the participants and who were invited to attend a focus group in one of the classrooms. Between three and six researchers were present during the data collection. The parents and teachers were allowed to stay in the classroom if they wanted to, but they were asked to refrain from providing answers on behalf of the children.

\subsection{Participant Selection}

A convenience sample of 19 typically developed children between 5 and 11 years of age who wanted to participate in an in-person meeting to draw and talk about robots and whose parents agreed with their child participating were included. The recruitment strategies included advertising with posters and through word-of-mouth. The recruitment finished with sampling saturation.

\subsection{Data Collection Procedures}

The study protocol was approved by the Ethics Research Board at the Universidad del Rosario, located in Bogotá, Colombia. We conducted four focus groups during two different data collection sessions. Two focus groups were conducted in the urban setting ( 9 children) and two in the rural setting (10 children). In each setting, the first focus group was attended by the youngest children (5-8 years of age) and the second focus group was attended by the oldest children (9-11 years of age). The first data collection session was conducted on 8, August, 2016, and the second on 19, August, 2016. The parents agreed to their children participating in a 2-h focus group. After obtaining informed consent from the parents and assent from the children, we invited the children to join the activity.

Each focus group was divided into three stages. During the first stage (asking), some questions were asked to the group of children in order to explore their knowledge of and previous experience with robots. The specific questions asked are presented in Table 1. Each question was read aloud and the children were encouraged to participate. During the second stage of the session (drawing), each child was asked to draw a robot for playing with. The research team provided the children with paper, pencils, colored pencils, and erasers. In order to encourage the children to draw a robot, we used the questions presented in Table 1 . We gave the children between 20 and 30 min to draw a robot. During the third 
Fig. 1 Data collection procedure

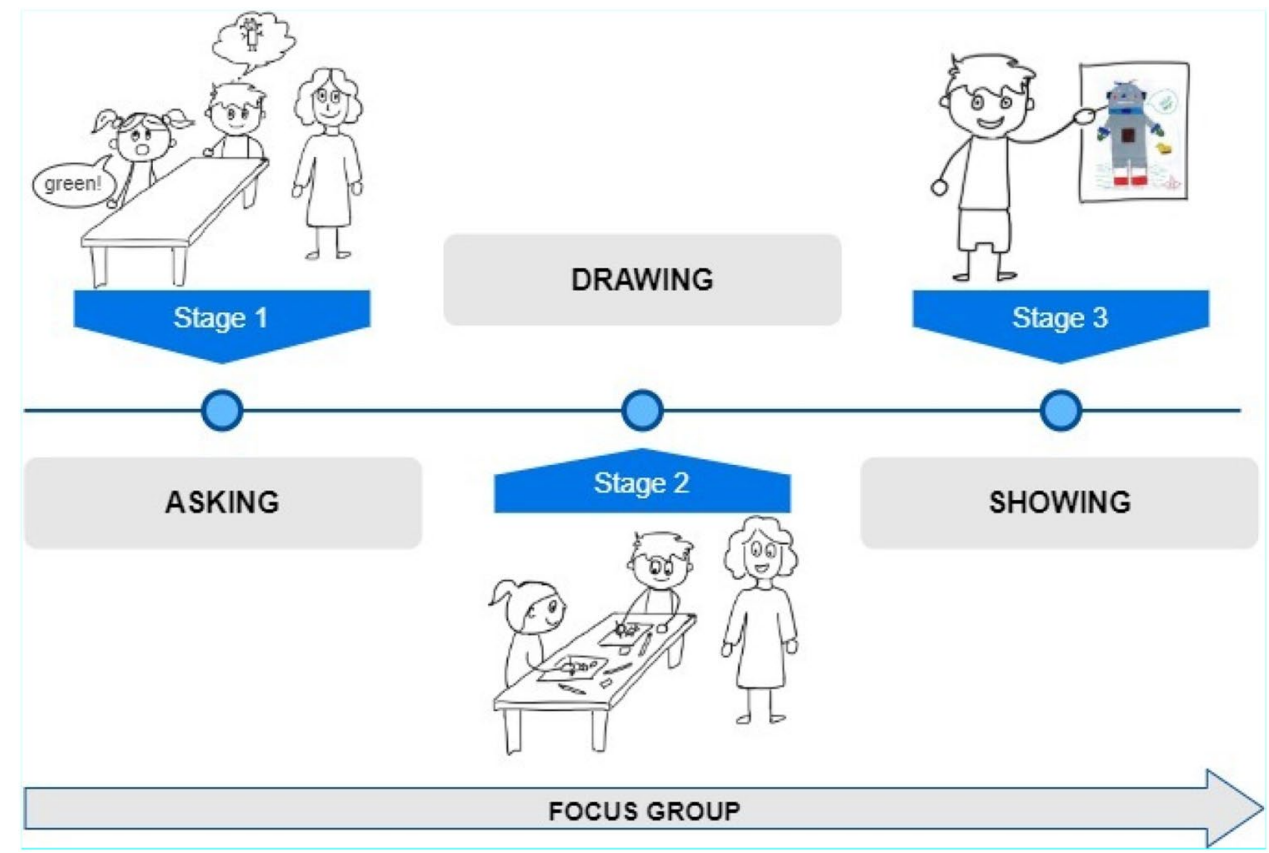

stage of the session (showing), after the children had drawn their robots, they were asked to describe their drawings and to answer the questions presented in Table 1 . We video and audio recorded the participants' descriptions and took pictures of the children's drawings. Table 1 and Fig. 1 depict the previously described process.

An analysis of the first two focus groups (first date) showed that the participants were mostly boys and that the topics of both the drawings and descriptions were influenced by commercially available robots or popular robots on television shows (e.g. Transformers). All the children were from middle-to-high socioeconomic strata. Thus, the research team decided that representations from girls, lower socioeconomic strata, and children who had been less exposed to commercially available robotic toys were needed. Then, the second session was conducted in the rural school located in the small town. The same data collection procedure was followed in each focus group.

\subsection{Data Processing and Analysis}

The unit of analysis was each child's drawing and description. The data were coded and analyzed using a summative approach to content analysis [26]. This approach is used when the researcher is interested in "identifying and quantifying certain words or content in text with the purpose of understanding the contextual use of the words or content" [26, p. 1283]. In this approach, a quantification of a particular content (e.g. frequencies of a word) is utilized to explore usage, which is followed by a latent content analysis or the interpretation of the appearance of a particular content. In order to follow this summative approach to content analysis we carried out the following steps. First, the participants' identifiers were replaced by codes. A file with the matches between the participants' names and codes was encrypted and protected with access only allowed by the principal investigator and one research assistant. Second, each focus group was individually transcribed by this research assistant using the participants' unique codes. Third, after reading all the data repeatedly, the research team agreed on the following categories of analysis; prior knowledge, previous experience, appearance, and functions. These categories were related to the questions we asked the children. The research team also identified two emerging categories, which are presented in the findings section. Fourth, based on these categories, two researchers identified and labeled every word related to each category using RQDA software (RQDA: R-based Qualitative Data Analysis) Version $0.3-1,{ }^{1}$ which was run on R Studio, ${ }^{2}$ Version 1.1.383, R version 3.4.3. Any text that could not be categorized with the initially defined categories was given a new code. Fifth, all the identified words were then exported to RQDA as an html-type file. At this step, we "cleaned up" the data by deleting accent signs (used in Spanish), blanks, and articles, and by replacing all capital letters with lower case letters. Sixth, the html files were manually transformed to csv format and uploaded again to the R Studio software to create word clouds based on the frequency of each word per category. The following

\footnotetext{
1 http://rqda.r-forge.r-project.org

2 https://rstudio.com/
} 
libraries were used at this last step: TM (Text Mining Package), Word Cloud, SnowballC, and GGplot 2. Additionally, for publication purposes two researchers proficient in Spanish and English translated the words from Spanish to English, and another researcher, also proficient in Spanish and English, reviewed the translations. Word cloud software generated a graph where the size of a word represented the frequency that word occurred in the text. After creating the word clouds, the research team discussed the frequency results based on the characteristics of the speakers in terms of gender, age, and social context (urban and rural).

To analyze the drawings we followed the next steps. First, the participants' identifiers were replaced by codes in each drawing file created. Second, two research assistants described each drawing and created a word file with a detailed description based on the observation of each drawing. These files plus the actual drawing were used to extract the main characteristics of each drawing.

Trustworthiness was judged using the criteria of credibility, transferability, dependability, and confirmability [27]. Credibility was achieved through engagement in the settings and immersion in the data. In this study, this was achieved by all the researchers being involved in the data collection as well as by transcribing each focus group. Also, the research team met every week to discuss the labels per category and content that could not be categorized with the initially defined categories. Transferability was achieved through the "applicability of the findings to other settings" [28], and was achieved by having a detailed description of the settings and participants [28]. A detailed description of the settings and participants can be found in the findings section. Dependability was achieved by having records that reviewed how decisions were made throughout the research. Confirmability was achieved when the findings could be confirmed by others. Both dependability and confirmability were achieved through the use of an audit trail [28]. Dependability and confirmability were achieved by documenting the research meetings where the research activities were planned, the data were discussed, and decisions were made, as well as by organizing the research files and sharing them with the research team.

\section{Findings}

\subsection{Participant Characteristics}

Nineteen participants (ten boys) took part in the study. The average age was 8.3 years old $(\mathrm{SD}=1.8)$. Overall, $42.11 \%$ of the children were from low socioeconomic strata, while the remaining $57.89 \%$ were from middle $(26.31 \%)$ and high $(31.57 \%)$ socioeconomic strata. Nine children were from the urban group (middle socioeconomic strata, mean $=3.7$
Table 2 Participant and setting characteristics

\begin{tabular}{ll}
\hline Characteristics & Participants $(n=19)$ \\
\hline Age (years), mean (SD; min-max) & $8.1(1.7 ; 5-10)$ \\
Gender & $n(\%)$ \\
Male & $10(52.63)$ \\
Female & $9(47.4)$ \\
Socioeconomic strata & $n(\%)$ \\
Low & $8(42.11)$ \\
Middle & $5(26.31)$ \\
High & $6(31.57)$ \\
Setting & $n(\%)$ \\
Rural & $10(52.63)$ \\
Urban & $9(47.4)$ \\
\hline
\end{tabular}

$\mathrm{SD}=0.8$, mode $=4$ ) while ten belonged to the rural group (social economic strata mean $=1.4, \mathrm{SD}=0.84$, mode $=1$ ). $\mathrm{P} 5$ and P6, and P9 and P10 were siblings (see Table 2).

\subsection{A Robot is a Machine Like a Doll that Moves}

Figure 2 shows a word cloud of the children's imaginaries regarding their knowledge and previous experience of interacting with robots (i.e. their descriptions before drawing the robots). The children mostly associated robots with machines (37\%) and dolls (25\%). For the children, a robot should normally be able to play (12\%), walk (9\%), transform into other things (9\%), move (7\%), grab stuff (7\%), and speak (7\%). Interestingly, when the children talked about what tasks they would like to have a robot do, the words play (21\%) and help (15\%) were the most common features that the children mentioned.

The children's imaginary about what robot is seemed to be influenced mainly by age and previous experience. While in the urban environment many children had robot toys or had some hands-on experience with robots, in the rural environment the children's previous experience with robots was from what they had seen in movies and on television shows. In the urban environment the younger children who had robot toys at home tended to describe a robot as something that transforms into another thing. For example, P3 said "[a robot] is a doll that transforms into a truck" (5-year-old boy, urban) or "it [a robot] transforms into a 'bot', or a dinosaur, or a vehicle" (P4, 5-year-old boy, urban). This description of a robot recalls the popular Transformers from the movies and toys. The older children in both the urban and rural environments were more open about their ideas about what a robot is. Some of the children from the urban environment emphasized their technical features. P8 (10-year-old boy, urban) said "[a robot] is something made with electricity that can do things, for you or for me, independent things, [a robot] has no life, well it has artificial life"; or P10 


\section{What is it?}

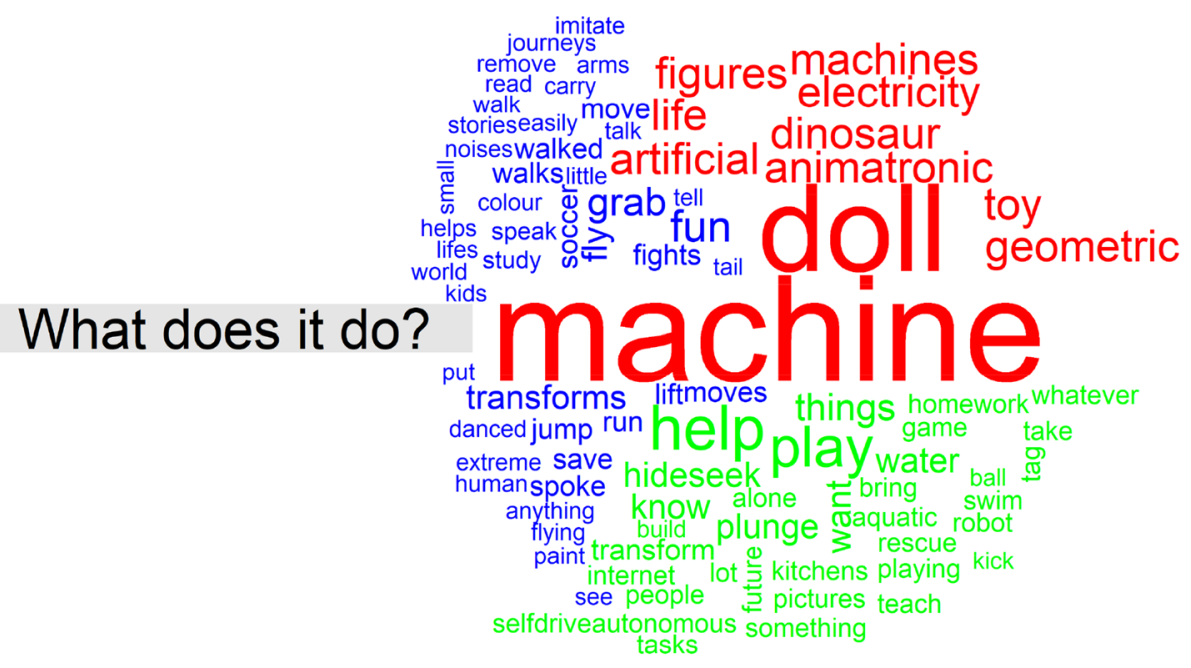

\section{What would you like it to do?}

Fig. 2 Knowledge and previous experience: What is a robot and what does a robot do?

(10-year-old girl, urban) said " it [a robot] is a machine like a doll that moves to play"; P16 (9-year-old girl, rural) said "a robot is like a machine that can do things." The children's imaginary of what a robot is did not seem to be influenced by gender or socioeconomic strata.

\subsection{A Robot Should be Humanoid, Tough, and Strong}

Figure 3 shows a word cloud of the children's imaginations (i.e. their descriptions after drawing the robot) regarding the category appearance. The children commented on a robot's appearance in terms of its material, shape, size, and controls. They emphasized that a robot should be made of metal (47\%); the metals named by the participants were metals in general (22\%), gold (13\%), silver (6\%), aluminum (3\%), and iron (3\%). Other materials were plastic (6\%) or concrete (6\%). But, more importantly, the children believed a robot should be tough and strong (22\%). Another aspect to highlight is that the children did not conceive a robot without controls, as they mentioned that a robot should have controls including buttons (52\%), a remote control (14\%), or a keyboard (5\%), or that it should be controlled via a cellphone (5\%). The analysis of the drawings revealed that, in terms of a robot's shape, $90 \%$ of the children drew a human-like robot, with legs and eyes. Other common elements of the robots were non-articulated arms (75\%), grips (45\%), hands (35\%), and antennas (55\%). To color in their robots the children mainly used blue $(21 \%)$, grey $(17 \%)$, and green $(13 \%)$ as the predominant colors. When explaining their drawings, the children said that these colors should be bright or shiny (18.18\%).

The girls created more defined drawings than the boys. The girls included a lot of details associated with a robot's functions. For example, P20 (9-year-old girl, rural) said "I press here [pointing a button] and it [the robot] can fly, and here [another button] and it [the robot] can dance." The girls tended to locate their robots' controls in the robot itself in the form of buttons, on/off switches, or keyboards. It was necessary to take into account the fact that there were no girls younger than nine years of age in our sample, so age could also explain why the girls were more detailed in their drawings than the boys.

A robot's appearance seemed to be influenced by the environment in which the children lived. All the children in the rural environment in both focus groups drew human-like robots with a head with two eyes and a mouth, two arms with hands and fingers, and two legs. $20 \%$ of the children in the urban setting drew animal-like robots, i.e. a spider or a biped fox. About $70 \%$ of the robots in the urban environment had neutral expressions, while neutral expressions were observed in about $40 \%$ of the robots drawn by the children in the rural 
Fig. 3 Appearance: What would the robot I want to play with look like?

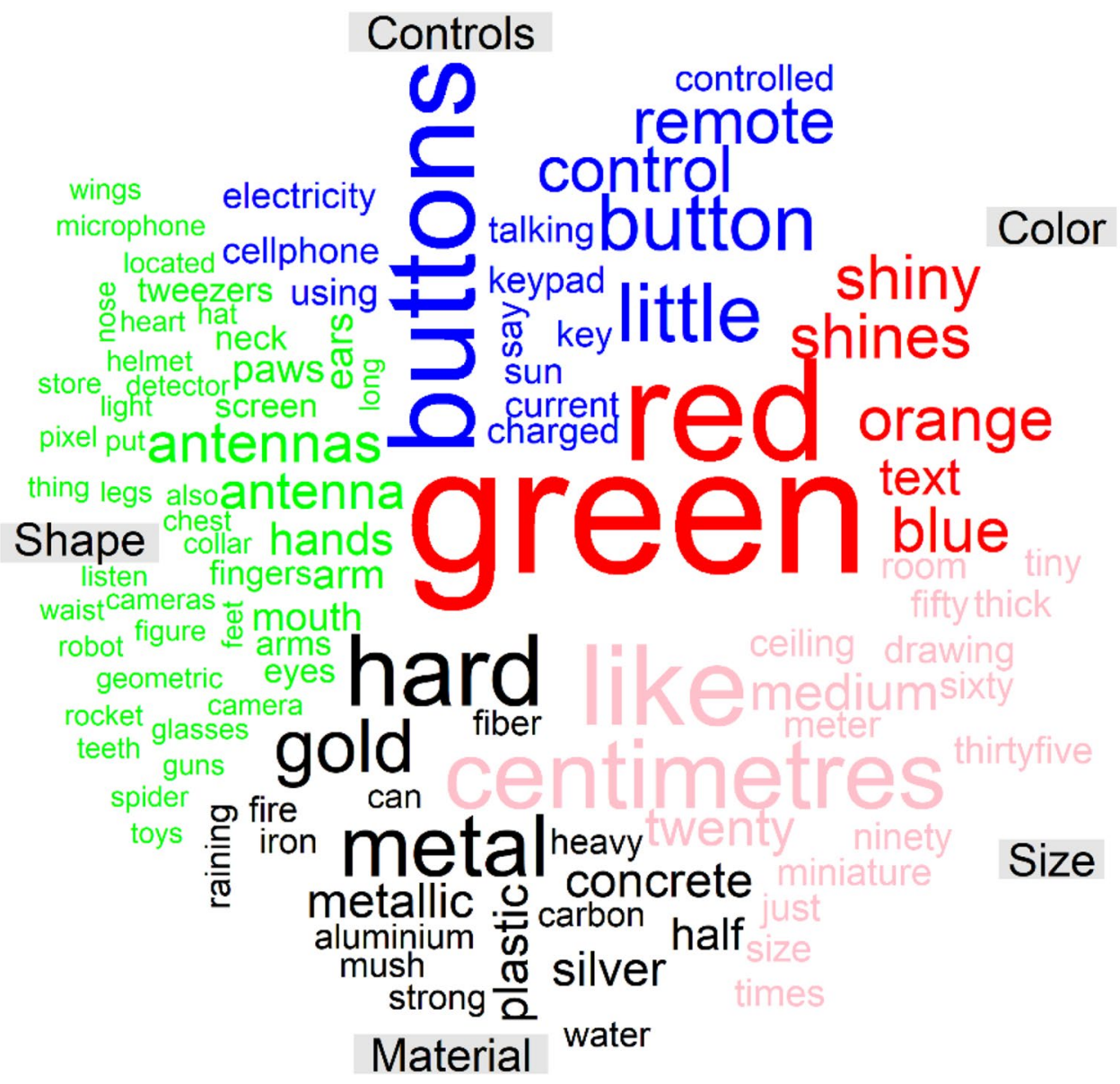

Fig. 4 Functions: What would the robot I want to play with do?

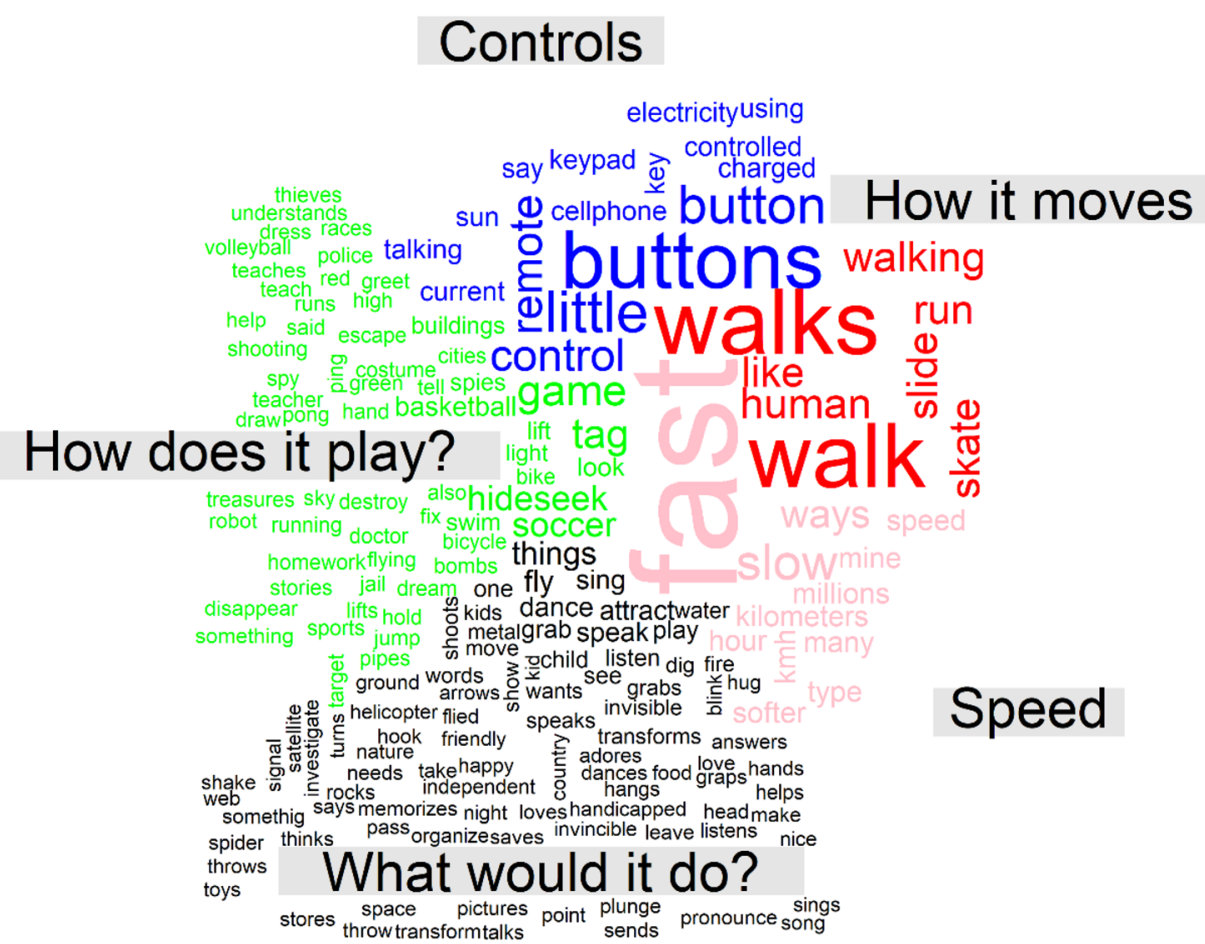


Fig. 5 Synthesis of the findings from the drawings

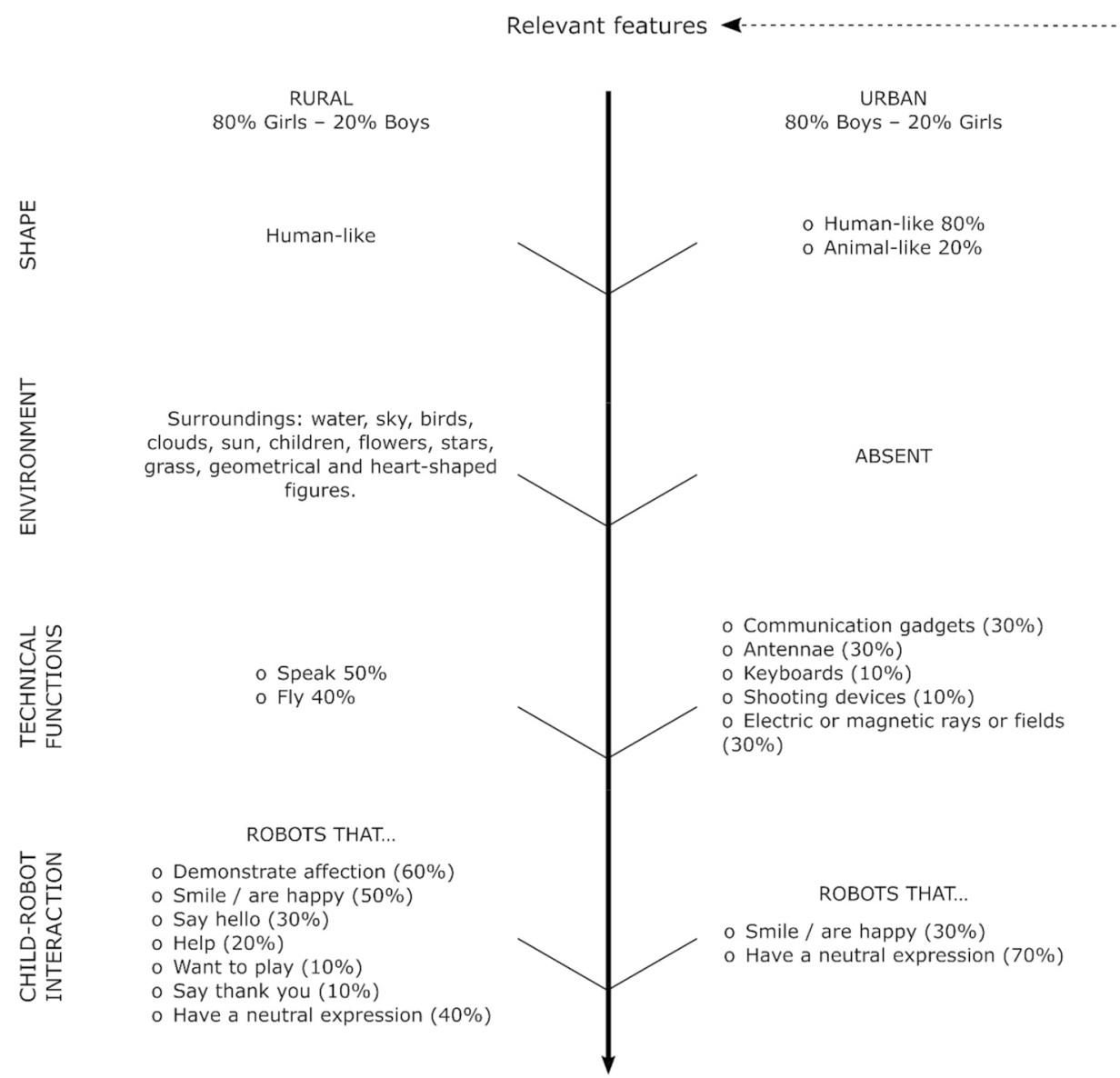

What would you like a robot to be like?

Is there anything you would like a robot to have? Is there anything you would like a robot to do? setting. Six children, 30\%, drew noses on their robots, all in the rural setting. In addition, two girls in the rural setting drew heart-shaped buttons on their robots' bodies.

\subsection{A Robot Should Walk, Grab Stuff, and Speak}

Figure 4 depicts a word cloud of how the children imagined the functions the robots they drew should perform. The children commented on how a robot should move, how fast it should be (i.e. speed), the functions associated with its controls, and the games a robot should play. Many of the children commented that a robot should move by walking (70\%) and be fast (60\%). The children mentioned a variety of games a robot should be able to play including tag (15\%), hide and seek (12\%), soccer (12\%), and basketball $(6 \%)$. Many of the children associated buttons with the functions a robot should perform such as flying $(8 \%)$, grabbing stuff (7\%), speaking (7\%), dancing (7\%), singing (7\%), listening (4\%), and transforming (3\%). The drawings corroborated what the children imagined a robot should do, i.e. a robot should be able to speak (25\%), fly (20\%), and shoot objects and fire $(5 \%)$.

The robots' functions seemed to be influenced by the environment. The children in the urban environment tended to include in their drawings elements related to technological features such as communications gadgets $(30 \%)$, antennae $(30 \%)$, electric or magnetic rays or fields $(30 \%)$, shooting devices (10\%), and keyboards (10\%). In contrast, the children from the rural environment included elements related to a robot having the capacity to speak (50\%) and fly $(40 \%)$. Interestingly, for the children in the urban setting the functions of the antennae were related to communication between a robot and its controls, while for the children in the rural setting their function was communication between child and robot. For example, P18 (9-year-old girl, rural) said "my robot listens to what children say through the antennae and he translates it."

The analysis of the narrative content and the drawings revealed two emergent main themes. Human-robot interaction from a child's perspective and robots within an 
environment. A synthesis of the findings from the drawings is presented in Fig. 5.

\subsection{Human-Robot Interaction From a Child's Perspective}

The children expressed ideas about how robots and humans interact. There were three common elements; robots express emotions, robots help people, and robots are companions.

\subsubsection{Robots Express Emotions}

From a child's perspective, emotions mediate human-robot interactions. An analysis revealed that emotions as mediators of child-robot interactions occurred mainly in the girls in the rural environment. The children perceived that robots should have the capability to demonstrate and communicate positive feelings; in some cases these feelings were toward the children. For example, when describing her drawing, P15 (9-year-old girl, rural) said:

"... it [pointing to an antenna] moves when it [the robot] is happy"... "there are little buttons, they are heart shaped so the child can see the feelings the robot feels for the child...that it [the robot] loves him."

P14 (9 year-old, girl, rural) said "he [the robot] is friendly." P18 (9 year-old, girl, rural) said "so he [the robot] says nice things." The drawings showed a lot of elements related to the emotions that a robot can demonstrate toward children. In $80 \%$ of the drawings done by the children from the rural environment, elements related to positive emotions were present. Smiling or cheerful robots were in $50 \%$ of the drawings, messages of greeting said by the robots were in $30 \%$ of the drawings (e.g. "hello friends"), demonstration of affection were in $60 \%$ of the drawings (e.g. heart shapes around the robot), an intention to play with the child were in $10 \%$ of the drawings (e.g. I want to play), and appreciation for the child were in $10 \%$ of the drawings (e.g. "you are the best"). In the urban environment $30 \%$ of the drawings showed a smiling robot.

\subsubsection{Robots Help People}

The children also thought that robots help people. Although this theme was common in both settings where the focus groups were conducted, the connotations of "help" were different between the settings. For the children in the urban area, robots can mainly help children with their schoolwork or household chores. For example, P8 (10-year-old boy, urban) said "[the robot] helps you with your homework;" and P9 (9-year-old boy, urban) said "they [robots] can help you with painting, coloring, and doing some assignments that you don't know how to do." For the children in the rural area, robots help other people who are in need. For example, P13 (10-year-old boy, rural) said "[a robot] will help a blind man;" P16 (9-year-old girl, rural) said "[a robot] helps someone walk or someone who was going to drown;" P18 (9-year-old girl, rural) said "for example, someone who was in a place where he could not find the exit and then the robot could fly and locate him and then he could reach the exit;" and P14 (9-year-old girl, rural) said "he [the robot] helps people ... who are lost and who are blind; and those who are sick."

\subsubsection{Robots are Companions}

In the rural environment in particular, the children tended to speak more about robots as companions than the children in the urban environment. For example, when asked what they liked most about robots, two girls (both 9 years old) from the rural environment highlighted that robots can play with them when nobody else is available to play. For example, “... when one does not have a brother, like me" (P18), or "when your mom cannot play with you" (P16). In the urban setting, P8 (10-year-old boy, urban) said "they [robots] imitate being your friend, tell you stories."

\subsection{Robots Within an Environment}

A common difference between the drawings done by the children in the urban setting and those in the rural setting was the environment where the robots were located. While the robots drawn by the children in the urban setting were in an empty space, i.e. there was no environment surrounding the robots, $90 \%$ of the children in the rural setting drew their robots in an environment that included water, the sky, birds, clouds, the sun, children, flowers, heart-shaped figures, stars, grass, and geometric figures. In $80 \%$ of the drawings the surroundings were nature. It was also interesting that the children in the rural environment not only tended to draw their robots located in a physical environment but also in a situational environment. That is, there was a situation or a problem that the robot had to solve, or a story was told in the drawing. For example, P14 (9-year-old girl, rural) drew her robot next to a girl who was crying and the robots asked "Are you lost? Yes/No," and the girl answered "Yes"; and P13 (10-year-old boy, rural) drew a robot holding the hand of a child who was next to a river. Figure 6 ( $a$ and $b$ ) shows two drawings done by the children.

\section{Discussion}

This study aimed to find out how typically developing children imagine what a robot is and what features would make a robot good for playing with. The results of this study provide 
Fig. 6 Examples of robots drawn by the children. a Robot drawn by P10, 10-year-old girl, urban setting. b Robot drawn by P13, 10-year-old boy, rural setting

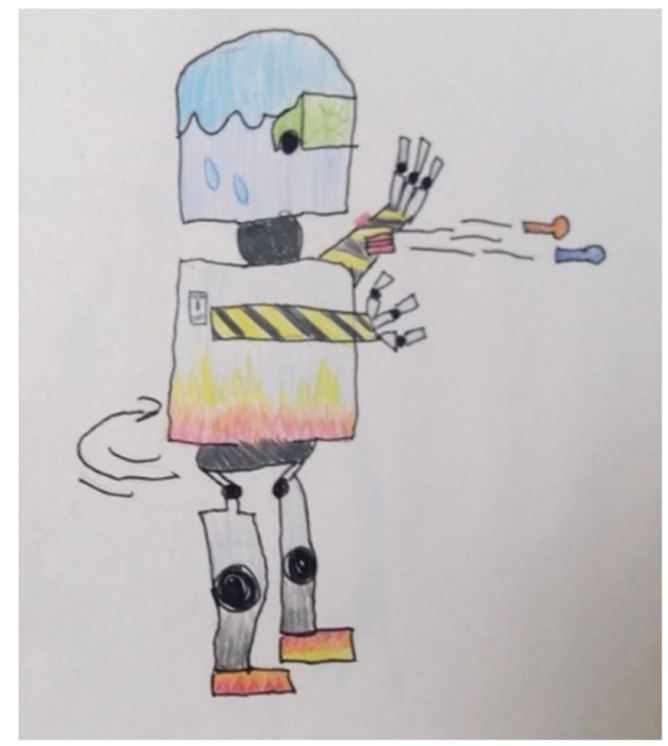

(a)

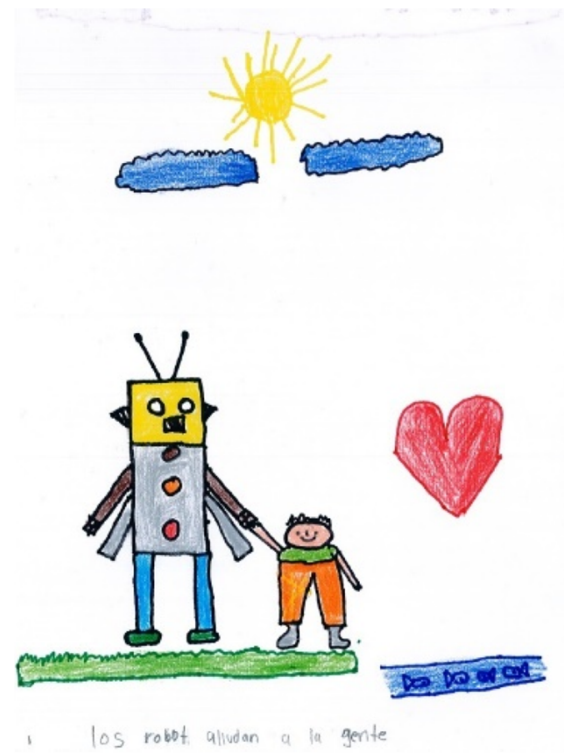

(b) valuable information about what children with sufficient play experience expect from a robot for playing with. The findings revealed that children as young as 5 years of age have an idea of what a robot is. The findings also revealed that children imagine that a robot good for playing with is one that has an anthropomorphic appearance, is tough and strong, has controls, and that is able to move, grab, speak, and play popular kids' games. Two new themes emerged from the analysis, the first related to human-robot interaction from a child's perspective, and the second to how children perceive robots within an environment. Since the authors' main purpose is to use the results of this study to design a robot that allows children with motor impairments to engage in play activities, part of the discussion will be dedicated to the implications of our findings in relation to this purpose.

The children in this study associated robots with machines and dolls. Most of the children represented this idea in the form of an anthropomorphic biped robot with the capacity to do things such as move, grab, speak, and listen. This shows that when asked what a robot is and to "create" a robot, most of the children thought about social robots. According to Breazeal [29], social robots are ones that people anthropomorphize in order to interact with them. We also observed that the children clearly differentiated between humans and robots. Two children (P13 and P14) drew a robot interacting with a child, with their drawings showing clear differences between the appearance of the robot and that of the child. Children with cerebral palsy have shown playfulness and engagement in play activities facilitated by several animal-like and machine-like robotic toys (Air Swimmer, Cubelets, Dash and Dot, Edison and Zoomer) [9], a socially assistive robot [11], ZORA (a version of the NAO robot with software developed for the field of care) [30], and an adapted car-like Lego robot [7]. To date, no studies have compared the acceptance of robots with different appearances (e.g. human-like, animal-like, and machine-like) by children with cerebral palsy. Children with cerebral palsy who used a car-like Lego ${ }^{\mathrm{TM}}$ robot for play referred to the robot as a "car" rather than a "robot" in their initial interactions with the robot; after a few sessions, they learned that that object was a robot and some incorporated the word "robot" into their vocabulary repertoire [7]. Thus, children's preferences remain unknown as the robots used and their features have been selected by other "experts" such as therapists or researchers rather than by the children. Considering that children with cerebral palsy want to play in the same way as typically developing children, but do not have the motor skills to perform the play activities, our results will inform researchers and developers about the features a group of typically developing children would prefer in a robot for playing with.

The children also identified that a good robot should be tough and strong. When asked about what materials the robots they had drawn were made of, the most common ones mentioned were metal (e.g. silver and gold) or concrete. The results indicate that a valuable feature for a robot is that it is strong enough to play games without falling apart. It seems that robots falling apart is a problem that children encounter when playing with them. For example, one study reported that when a Lego ${ }^{\mathrm{TM}}$ robot was adapted so that children with severe cerebral palsy could control it during free play activities, during some play sessions the children became frustrated as the robot fell apart when they wanted to grasp, push, or carry some objects that were heavier or bigger than the robot [7]. Many robots that have been used by children with disabilities are fragile, small, or do not have 
the capacity to grab objects, which may limit the children's play experience [6]. In this respect, typically developing children help us to confirm that for children with and without disabilities it is important that a robot for playing with is tough and strong enough for them to be able to freely explore their environment during play activities.

The children identified that a robot should be able to move around, grab objects, speak, and play popular kids' games from the culture the children were immersed in. These games included hide and seek, tag, soccer, and basketball, and are popular in the context in which this study was conducted. For example, soccer is the national sport in Colombia; thus, it is common that children from this culture play and enjoy soccer. Despite play being an occupation children from all cultures engage in, there is a huge variety of play themes and activities within and across cultures [31]. The sociocultural theories of play state that play is a concept related to social life and culture; thus, play is influenced by culture, which means that play themes and games are based on cultural material [1]. Children from other geographical areas who grow up in other cultures may have different preferences about the play activities and games a robot should perform. The games that the children in our study wanted a robot to play were also influenced by the developmental stages of play. Piaget was the first author to state that play follows developmental stages that are related to the progression of intelligence. These stages are known as Piaget's classification of games. The first stage is functional play, which begins during the first month and lasts until the age of two years. The second stage is symbolic play, which typically occurs between two and seven years of age. The third stage is games with rules, which begins at around seven years of age; this stage is very advanced by about eleven years of age, after which it becomes the kind of play engaged in by adults. This kind of play mostly comprises games where social interaction is the main element [32]. Most of the children who participated in our study were at the developmental stage of games with rules (Age mode $=9$ years old), which explains the games the children wanted the robot to play. In summary, the developmental stage and culture are aspects that should be taken into account by developers and researchers in order to design developmentally and culturally appropriate robot hardware and programs, as well as play activities where robots are used.

We noticed that the children from the urban setting talked about and drew the technical features of a robot in more detail than the children in the rural setting. This could be explained by differences in the children's level of exposure to the technical aspects of robots, for example, participating in robot summer camps or robot workshops, or having a parent who is an engineer. Access to this kind of experience may be limited in rural settings due to several factors in the Colombian context. It has been reported that access to high-quality education is limited in rural settings [33], that family income in urban settings is significantly higher than in rural families, and that the level of education is $56 \%$ lower for rural men and $49 \%$ for rural women than that for people living in cities [34]. Another explanation for the technical details provided by the children in the urban setting might be what Søraa and colleagues [15] called "technological determinism." How a child imagines what a robot is and does is based on learned experience predetermined by exposure to existing robots. With less exposure to commercially available robots, the children in the rural setting were less prone to be influenced by technological determinism. Including children with less exposure to what adults have established as "a robot" could help to shift the paradigm from children adapting to robots based on adults imaginaries, in the sense described in [20], to shaping social robots based on children's imaginaries, which is an emergent approach [15, 17].

The children highlighted communication with a robot as being an important feature. The means of communication included operating the robot using buttons located in the robot itself, or by remote control, or using verbal commands. The children in the rural setting established the main function of a robot antenna as listening to what the child says. The children also wanted a robot to be able to speak. Thus, some of the children imagined natural communication with their robots.

The emergent themes from this study provide valuable information about how children perceive robots. With regard to human-robot interaction, children had the imaginaries that robots should be able to express emotions, mainly positive ones, toward children. The girls in our sample commented on and drew their robots saying nice things to children and making children feel appreciated. One girl suggested in her drawing that the robot recognized that a girl was lost and sad (she was crying), and that it was willing to help. Thus, from a child's perspective it is important that a robot is able to express emotions, mainly by talking to the child. The children also perceived a robot as being a companion. These findings tell us that the social imaginary of a robot from a child's perspective is guided, as pointed out by Søraa and colleagues, by an emotional connection with the robot, rather than by a rationally driven notion of the robot's usefulness [15]. Our results are also consistent with [16], who found that from a child's perspective robots are entities with which children can form social relationships. Robots' characteristics and styles of interaction influence the levels of engagement and enjoyment children expect to find when interacting with robots, which is critical for the formation of child-robot interaction. A considerable amount of research has been dedicated to developing social robots that are able to recognize and react to human emotions using artificial intelligence $[6,11]$. Thus, this feature is being sought by developers. 
The children also had the imaginary that robots help people. For the children in the rural setting, functions such as flying, listening, and speaking were associated with helping someone in trouble or who needs help. These functions described by the children went beyond robots built just for playing with and moved toward those with "serious" functions such as helping someone who is lost or has a disability. There is abundant research showing that women are better at expressing feelings toward another person who may be in trouble or in need than men. In fact, girls from low-income families in Colombia, Argentina, and Spain have shown higher levels of prosocial behaviour and empathy than boys [35]. Thus, our findings may have been due to the fact that the focus groups in the rural setting comprised mainly girls. The small number of boys in the rural focus groups might have been influenced by the girls in their descriptions and drawings, thus resulting in the predominant idea that robots that help people come from rural environments.

The children in the rural setting located their robots in environments that were mainly composed of natural elements. This could be explained by previous research that found that children living in rural areas perceive nature as a living presence more than children from cities [36]. Similarly, in our study most of the children from the rural setting drew their robots surrounded by nature. One participant dedicated about $80 \%$ of the time to drawing nature while spending only a few minutes at the end of the session drawing the robot. This may have been because children from rural areas feel they belong in nature. In contrast, it is well known that children in cities spend less time in outdoor environments and interacting with nature, and that the time they spend indoors using electronic devices is increasing [36]. Thus, the children from the rural setting were shown to be more aware of the natural environment they are immersed in, while the children from the urban setting were shown to be less aware of their environment and more aware of the robots' technical features.

\subsection{Recommendations for Developers}

Based on our results, we can provide the following recommendations to researchers, clinicians, or entrepreneurs who want to develop robotic systems for promoting play in children with and without disabilities.

- The robot's appearance matters to children. Anthropomorphic robots that are clearly machines rather than humans might be more accepted by children with and without disabilities.

- Children want to control the robot through some means whereby they communicate with it. Developers could explore verbal commands as a natural way of controlling robots. This could be a consideration for children with motor impairments such as cerebral palsy, as many of them have very limited spoken language due to their motor impairments, thus making it difficult to control robots through verbal commands. However, children with motor impairments that do not affect communication would be able to use verbal commands.

- Children want a robot for playing with to be able to move, grab, speak, and play popular kids' games from the culture they are immersed in and according to their developmental stage.

- A good robot should be tough and strong so it is able to interact with diverse objects in the environment. Thus, the materials used in robot design must be resistant, durable, and facilitate the placement of accessories for performing or pretending to do play activities. For example, wings for "flying," antennae for communication, grips or "hands" to grasp objects, and lights and sounds that provide feedback to the child, e.g. lights that let the child know that the robot has received a command and is ready to run a program.

- Developing low-cost robotic systems makes them affordable for children in low income settings. Some of the features desired by children can be expensive. For example, robots that are able to recognize emotions are usually expensive and may not be affordable for children in low income settings. Alternatives for low cost robots could be the creation of pre-stored positive messages that children can play back or that are configured to be played at a specific time (e.g. 4:30 pm on week days), for example saying to the child "I missed you, do you want to play?" when the child gets home from school.

- From a child's perspective, child-robot interaction is more emotional than rational, so robots should have characteristics that facilitate these emotional relationships. This is especially important for girls in some cultures. Asking children with low levels of exposure to commercial robots about the features a robot should have might shift the paradigm from technological determinism and established ideas of robots as nurtured by commercial companies and movie corporations to social constructivism based on children's imaginations, wishes, and dreams.

\subsection{Limitations}

This study has some limitations. The first might consist of the purposeful and snowball sampling method. However, the data collection and analysis supported data saturation, and the researchers made adjustments to the data collection in order to achieve variation in the samples in the two different settings. In addition, we conducted four focus groups, and research has found that conducting three focus groups is enough to reveal more than $90 \%$ of all themes in qualitative research 
[37]. A second limitation might be that two pairs of siblings participated in the same group from the urban focus groups. The two siblings wanted to be seated at the same table during the drawing activity. Their drawings were almost identical, so one must have influenced the other. Third, this study's results reflect the imaginaries of a small group of children from both a rural and an urban setting in a developing country. However, the researchers did their best to achieve variation in terms of gender, age, socioeconomic strata, and setting.

\section{Conclusion}

The results of this study will inform developers and researchers about the features that make a robot good to play with from the perspective of typically developing children with sufficient play experience. Children's imaginaries about good robots for playing with include those with an anthropomorphic appearance, that are tough and strong, that have controls, and that are able to move, grab, speak, and play popular kids' games. Age, gender, culture, and the physical environment in which the children lived influenced what the children expected to find in a robot for playing with during child-robot interactions. How the children imagined robots was also influenced by their exposure to popular robots available in the technology and movie industries. The girls expected that robots would be able to express emotions and help people, thus highlighting an emotional connection with robots in their imaginaries. As children with motor impairments want to play in the same way as typically developing children, even though their motor skills are compromised, developers and researchers can use the results of this study when creating or adapting robotic systems for children with motor impairments, as well as for children with other impairments or typically developing children.

\begin{abstract}
Acknowledgements We would like to thank the study's participants and their parents as well as the "Colegio Departamental Bagazal" and its Principal, Elsa Luzmila Heredia. We would also like to thank the research assistants, occupational therapy and biomedical engineering students, for their help in conducting this study. We would also like to thank the psychologist Mariana Andrea Pinillos Guzmán for her help in administering the assent to the participants.
\end{abstract}

Funding (information that explains whether and by whom the research was supported) This project was funded by the "Fondo de Investigación Universidad del Rosario (FIUR/2016)" grant number: FUIR QDNBG007.

\section{Declarations}

Consent to participate (include appropriate statements) Written informed consent was obtained from the parents.

Consent for publication (include appropriate statements) The parents provided written consent for the children's drawings to be published in a scientific journal. The authors affirm that the human research participants provided informed consent for the publication of the images in Fig. 6a and b.

Ethical approval This study was performed in line with the Declaration of Helsinki principles. Approval was granted by the Ethics Committee of the Universidad del Rosario (April 26, 2016/No. CEIABN026-000131).

Open Access This article is licensed under a Creative Commons Attribution 4.0 International License, which permits use, sharing, adaptation, distribution and reproduction in any medium or format, as long as you give appropriate credit to the original author(s) and the source, provide a link to the Creative Commons licence, and indicate if changes were made. The images or other third party material in this article are included in the article's Creative Commons licence, unless indicated otherwise in a credit line to the material. If material is not included in the article's Creative Commons licence and your intended use is not permitted by statutory regulation or exceeds the permitted use, you will need to obtain permission directly from the copyright holder. To view a copy of this licence, visit http://creativecommons.org/licenses/by/4.0/.

\section{References}

1. Parham LD (2008) Play in occupational therapy. In: Parham LD, Fazio LS (eds) Play in occupational therapy for children. Mosby Elseiver, St. Louis, pp 3-39

2. Ferland F (2005) The Ludic model, 2nd edn. CAOT publications ACE, Ottawa (Ontario)

3. Lindsay S, Rampertab L, Curran C (2019) Therapy through play: advancing the role of Robotics in paediatric rehabilitation. In: EverydayTechnologies in Healthcare. CRC Press, Boca Raton. Taylor and Francys Groups, pp 11-29.

4. Chang HJ, Chiarello L, Palisano R, Orlin M, Bundy A, Gracely E (2014) The determinants of self-determined behaviors of young children with cerebral palsy. Res Dev Disabil 35:99-109

5. van den Heuvel RJF, Lexis MAS, Gelderblom GJ, Jansens RML, de Witte LP (2016) Robots and ICT to support play in children with severe physical disabilities: a systematic review. Disabil Rehabil Assist Technol 11(2):103-116

6. Miguel Cruz A, Ríos Rincón AM, Rodríguez Dueñas WR, Quiroga Torres DA, Bohórquez Heredia AF (2017) What does the literature say about using robots on children with disabilities? Disabil Rehabil Assist Technol 12(5):429-440

7. Rios A, Adams K, Magill-Evans J, Cook A (2016) Playfulness in children with limited motor abilities when using a robot. Phys Occup Ther Pediatr 36(3):232-246

8. Rios Rincon AM. Playfulness in children with severe cerebral palsy when using a robot. Edmonton: University of Alberta, $\mathrm{PhD}$ dissertation; 2014.

9. Bulgarelli D, Bianquin N, Besio S, Molina P (2018) Children with cerebral Palsy playing with mainstream Robotic toys: playfulness and environmental supportiveness. Front Psychol 9(1814).

10. Mahdi H, Saleh S, Shariff O, Dautenhahn K. Creating MyJay (2020) A new design for Robot-assisted play for children with physical special needs. In: Wagner AR, Feil-Seifer D, Haring KS, Rossi S, Williams T, He H, Ge SS (eds) Social Robotics. ICSR 2020. Lecture Notes in Computer Science; 2020; Golden, pp 676-687.

11. Clark C, Sliker L, Sandstrum J, Burne B, Haggett V, Bodine C (2019) Development and Preliminary Investigation of a Semiautonomous Socially Assistive Robot (SAR) Designed to Elicit Communication, Motor Skills, Emotion, and Visual Regard 
(Engagement) from Young Children with Complex Cerebral Palsy: a Pilot Comparative Trial. Adv Human-Computer Interaction 2019:14

12. Patrizia M, Claudio M, Leonardo G, Alessandro P (2009) A robotic toy for children with special needs: From requirements to design. In: IEEE International Conference on Rehabilitation Robotics; Kyoto, Japan, pp 918-923.

13. Kronreif G, Kornfeld M, Prazac B, Mina S, Fürst M (2007) Robot assistance in playful environment - user trials and results. In: IEEE International Confernece on Robotics and Automation; 2007; Roma, Italy, pp 2898-2903.

14. de Jong C, Peter J, Kühne R, Barco A (2019) Children's acceptance of social robots A narrative review of the research 20002017. Interact Stud 20(3):393-425

15. Søraa RA, Nyvoll PS, Grønvik KB, Serrano JA (2020) Children's perceptions of social robots: a study of the robots Pepper, AV1 and Tessa at Norwegian research fairs. AI \& Soc.

16. van Straten CL, Peter J, Kühne R (2020) Child-robot relationship formation: a narrative review of empirical research. Int J Soc Robot 12:325-344

17. Vallès-Peris N, Angulo C, Domènech M (2018) Children's imaginaries of human-robot interaction in healthcare. Int J Environ Res Public Health 15(5):970

18. Sartre JP (2004) The imaginary A phenomenological psychology of the imagination. Routledge, London and New York

19. Marcus GE (ed) (1995) Technoscientific imaginaries: conversations, profiles, and memoirs. University of Chicago Press, Chicago and London

20. Šabanović S (2010) Robots in society, society in robots. Int J Soc Robot 2:439-450

21. Druin A (2002) The role of children in the design of new technology. Behav Inform Technol 21:1-25

22. Malinverni L, Valero C (2020) What is a robot?: an artistic approach to understand children's imaginaries about robots. In: Proceedings of the Interaction Design and Children Conference (IDC '20); New York, NY, pp 250-261.

23. Bradshaw C, Atkinson S, Doody O (2017) Employing a qualitative description approach in Health Care Research. Global Qualitative Nursing Research.

24. Wildemuth BM (1993) Post-positivist research: two examples of methodological pluralism. Library Quart Inform Community Policy 63(4):450-468

25. O'Brien BC, Harris IB, Beckman TJ, Reed DA, Cook DA (2014) Standards for reporting qualitative research: a synthesis of recommendations. Acad Med 89(9):1245-1251

26. Hsieh HF, Shannon SE (2005) Three approaches to qualitative content analysis. Qual Health Res 15(9):1277-1288

27. Lincoln YS, Guba EG (1985) Naturalistic inquiry. SAGE, Thousand Oaks.

28. Mayan MJ (2009) Essentials of qualitative inquiry. Routledge, New York

29. Breazeal C (2003) Toward sociable robots. Robot Auton Syst 42(3-4):167-175

30. van den Heuvel RJF, Lexis MAS, de Witte LP (2020) ZORA Robot based interventions to achieve therapeutic and educational goals in children with severe physical disabilities. Int $\mathrm{J}$ of Soc Robotics 12:493-504

31. Özdoğru AA (2019) Cross-cultural psychology of play and early childhood education. In: Association IRM. Early Childhood Development: Concepts, Methodologies, Tools, and Applications. IGI Global; pp 1-19.

32. Piaget J (1951) Play, dreams and imitation. Norton, New York

33. Carrero Arango ML, González Rodríguez MF (2017) La educación rural en Colombia: experiencias y perspectivas. Praxis Pedagógica 16(19):79-89
34. NU. CEPAL. Estudio Económico de América Latina y el Caribe 2002-2003. Santiago: CEPAL; 2003.

35. Mesurado B, Richaud MC, Mestre MV, Samper-García P, TurPorcar A, Morales Mesa SA, Francisco VE (2014) Parental expectations and prosocial behavior of adolescents from low-income backgrounds: a cross-cultural comparison between three countries-Argentina, Colombia, and Spain. J Cross Cult Psychol 45(9): 1471-1488

36. Profice C. Nature as a living presence: Drawings by Tupinambá and New York Children. PLOS ONE. 2018;13(10):e0203870.

37. Guest G, Namey E, McKenna K (2017) How many focus groups are enough? Building an evidence base for nonprobability sample sizes. Field Methods 29(1):3-22

Publisher's Note Springer Nature remains neutral with regard to jurisdictional claims in published maps and institutional affiliations.

Adriana Maria Ríos Rincón is an Assistant Professor whose research interests are focused on the role of assistive technologies in improving the levels of functioning, capacity, and participation in people living with limitations. Her research topics include the development of new low-cost, high-quality assistive technologies; and studying the effects of assistive technologies on the occupational performance, functioning levels, and social participation of people with disabilities. Her current research is devoted to investigating the use of assistive technologies to assess and promote cognitive skills and engagement in play in individuals with disabilities, mainly those with motor and cognitive impairments throughout their lifespans. She wants to increase understanding of how technology supports the health and wellness of individuals with disabilities through engagement in play. She was affiliated with the Universidad del Rosario at the time the project was conducted.

William Ricardo Rodríguez-Dueñas is a researcher and Associate Professor on the biomedical engineering program at the School of Medicine of Health Sciences, Universidad del Rosario. His background is in biomedical engineering (Bachelor's and Master's) and in 2010 he completed his $\mathrm{PhD}$ about speech technologies for children with communication disabilities. Currently, William is involved in research projects on assistive technology in care for elderly people and for children with disabilities.

Daniel Alejandro Quiroga Torres is a researcher and Professor on the biomedical engineering program at the School of Medicine and Health Sciences, Universidad del Rosario. His background is in biomedical engineering (Bachelor's), and he is doing a Master's degree in rehabilitation science. Currently, Daniel is involved in research projects on assistive technology for people with sensory disabilities and Internet of Things applications for health.

Andrés Felipe Bohórquez is a mechanical designer with a Bachelor's degree in mechanical engineering. He is currently working in the private sector on mechanical and automation projects. In 2016, he worked at the Universidad del Rosario as a research assistant in the biomedical engineering department.

Antonio Miguel-Cruz is an Adjunct Assistant Professor at the University of Waterloo and an assistant lecturer and clinical researcher at the University of Alberta and Glenrose Rehabilitation Hospital. He is an engineer whose research focuses on development and assessment, user adoption of technologies for rehabilitation assessments and interventions, and ways technologies can help people with disabilities, older adults, and family caregivers. He was affiliated with the Universidad del Rosario at the time the project was conducted. 